DISEASE PREVENTION है www.sciencemag.org/special/prevention

"black box" warning label.

The pendulum began to swing back in favor of aspirin, however, when Rothwell, a neurologist, noticed that fewer overall cancers had occurred among patients in the treatment arm of randomized trials launched in the 1980s to test aspirin as a way to prevent stroke. Amassing details on the cancers from patients' paper health records and U.K. health databases, his team initially confirmed aspirin's ability to ward off colorectal cancers, reporting in 2007 that there were $24 \%$ fewer cases and 35\% fewer deaths in the aspirin group after several years. Then in late 2010, they reported that patients taking aspirin at any dose daily for at least 5 years cut by $21 \%$ the long-term risk of dying from colorectal, gastrointestinal, esophageal, stomach, pancreatic, brain, lung, and prostate cancers.

Rothwell's group expanded on these results in three papers published in The Lancet and The Lancet Oncology this spring. One, a meta-analysis of 51 randomized trials in which aspirin was taken daily, found $37 \%$ fewer deaths from cancers after
Believer. Peter Rothwell's cancer studies convinced him to take a daily aspirin.

5 years. (The study came with a large caveat: It excluded WHS and an earlier trial that found no benefit, the Physicians' Health Study, because participants in each took aspirin only every other day.) This meta-analysis also found that while the aspirin group had more stomach bleeds, these incidents were not fatal-people recovered - and the bleeding risk went down after several years on aspirin. Another paper found that people on aspirin who developed cancer were $36 \%$ less likely to have tumors that had spread. A third paper found a "remarkable consistency" in the drop in cancers among aspirin users in epidemiologic studies and clinical trials, Rothwell says.

While these studies weren't designed specifically to test aspirin against cancer, some researchers say that because so many people now take aspirin off-label it would be diffi-

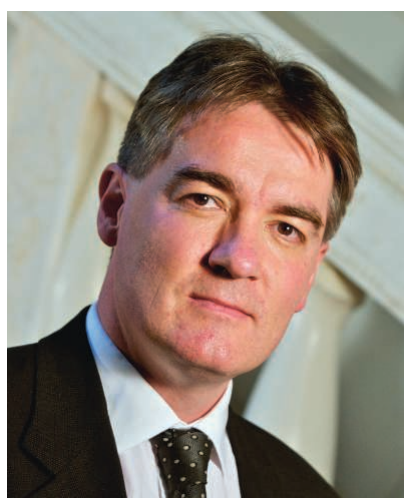

cult to conduct better trials. The Rothwell studies are "probably the best evidence we'll ever have on this topic," Chan says.

\section{Weighing risks}

Chan is part of an international panel on cancer prevention that, in response to the Rothwell studies, plans to update its stance on aspirin published 3 years ago. The group's leader, epidemiologist Jack Cuzick of Queen Mary, University of London expects the panel may suggest that people take low doses of aspirin daily starting around age 50 and stopping by age 70 , when the risk of internal bleeding rises. The group may also discuss whether doctors should screen patients for the ulcer-causing Helicobacter pylori bacterium, treating those who test positive with antibiotics before putting them on aspirin, to reduce the risk of bleeds.

Some U.S. researchers also suggest that it's time for the U.S. Preventive Services

\title{
Wondering How the Wonder Drug Works
}

HOW DOES TAKING ASPIRIN WARD OFF CANCER? RESEARCHERS still don't understand the mechanism, a lack of knowledge that threatens to frustrate development of better cancer prevention agents. But some say the latest clinical studies linking low-dose aspirin use with less cancer (see main story, p. 1471) suggest there's no need to improve upon this 113-year-old workhorse drug.

Among its many known effects, aspirin, or acetylsalicylic acid, inhibits two forms of enzymes known as cyclooxygenases (COX) that convert arachidonic acid into lipids called prostaglandins. Prostaglandins made by COX-1 protect the stomach lining, while those made

by COX-2 are involved in inflammation. Aspirin's inhibitor effects on COX-1 seem to explain why the medicine can upset stomachs and trigger internal bleeding. Many researchers have concluded that aspirin prevents cancer mainly by blocking the activity of COX-2. That's because the same inflammation-driven responses that help tissue recovery from wound injury-cell division, blood vessel formation, and suppression of programmed cell death, for example-may also help tumors grow.

Several lines of evidence implicate COX-2 in cancer induction. The enzyme is overproduced in many cancer types; mice lacking its gene are less prone to colon cancer; and trials of nonsteroidal anti-inflammatory drugs (NSAIDs) that target only COX-2 protected against precancerous colon polyps in people at high risk.

Reducing inflammation via COX-2 probably isn't the only way aspirin prevents cancer, however. "We are as a field getting down to some of the mechanisms, but it's very complicated," says Raymond Dubois of the MD Anderson Cancer Center in Houston, Texas. A decade ago, researchers found that aspirin can block production of a protein called
NF- $\kappa B$ that protects cells from early death. Although the studies were done in the test tube at aspirin doses much higher than those in the bloodstream, the NF- $\kappa$ B hypothesis remains on the table-along with more than a dozen possible mechanisms-from inhibiting various cell growth pathways to targeting cancer stem cells. "There are a number of very different, divergent ideas," says Asad Umar of the National Cancer Institute's Division of Cancer Prevention in Bethesda, Maryland.

Some experts say new clinical studies of low-dose aspirin and cancer suggest that COX-2 isn't directly involved at all. That's because at low doses, the drug doesn't block COX-2 but still impairs platelets, the small cell fragments in blood that help form clots, via the COX-1 pathway. Studies suggest that platelets blunt the immune attack on cancer cells traveling in blood and produce growth factors that help them take root in a new place, notes University of Pennsylvania pharmacologist Garrett FitzGerald. Some experiments suggest that activated platelets can also stimulate the COX-2 pathway in adjacent cells, which would explain how aspirin, by damping COX-2 at the site of an injury, could block the early stages of colorectal cancer, says Carlo Patrono, a pharmacologist at the Catholic University in Rome, Italy.

Efforts to make an alternative to standard aspirin haven't yet panned out. Several drugs that target only COX-2, notably Vioxx and Celebrex, unacceptably raised heart attack risk, for example. And a version of acetylsalicylic acid containing a chemical group called nitric oxide that was supposed to reduce side effects failed to gain regulatory approval. Some researchers are combining aspirin with other compounds such as a lipid to reduce stomach upset. But these are years from the clinic. For now, "I don't think a better aspirin is the issue," but rather figuring out which individuals stand to benefit from it most, FitzGerald says. -J.K. 